\title{
MCM4 in human hepatocellular carcinoma: a potent prognostic factor associated with cell proliferation
}

\author{
Yan Xu, Xueling Yang, Tongguo Si, Haipeng Yu, Yong Li, Wenge Xing, Zhi Guo* \\ Department of Interventional Therapy, Tianjin Medical University Cancer Institute and Hospital, National Clinical Research Center for Cancer, Key \\ Laboratory of Cancer Prevention and Therapy, Tianjin's Clinical Research Center for Cancer, Tianjin, China.
}

SUMMARY Hepatocellular carcinoma (HCC) remains a major public health problem. MCM4, a constitutive member of the minichromosomal maintenance protein family, has been reported to play a vital role in cancer malignancy behavior. However, the function of MCM4 in HCC remains largely unknown. The present study explored the specific role of MCM4 in HCC. The data from public datasets including TCGA and GTEx showed that MCM4 was overexpressed in HCC and significantly associated with poor prognosis. Immunohistochemistry results from $102 \mathrm{HCC}$ patients suggested that high-level expression of MCM4 was correlated with tumor size. Then a series of in vivo and in vitro experiments were performed to investigate the function of MCM4 in HCC tumor cells. MCM4 silencing suppressed the cell proliferation and sphere formation of hepatoma cells. Moreover, silencing MCM4 significantly decreased the growth of tumors in a xenograft tumor model. In conclusion, the results of the present study indicated that MCM4 was a potential prognostic predictor associated with poor outcomes of HCC patients and even a therapeutic target for HCC.

Keywords MCM4, minichromosomal maintenance protein family, hepatocellular carcinoma, prognostic marker, tumor proliferation

\section{Introduction}

The minichromosomal maintenance (MCM) protein family consists of six related proteins that have essential roles in the initiation of DNA replication, elongation of DNA replication, and other chromosome transactions (1-4). MCM4 is part of the MCM2-7 heterohexameric complex, that has ATPase activity and serves as the core of the replicative helicase that unwinds duplex DNA and drives the progression of the replication fork (5). It has been recently reported that deregulation of MCM4 can contribute to cell proliferation and tumorigenesis. Therefore, aberrant expression of MCM4 may be applied as a promising prognostic marker in several malignancies (4,6-10). However, there is still a shortage of studies exploring the correlation between MCM4 and HCC. The role of MCM4 in HCC remains unclear.

In this study, we systematically investigated the roles of MCM4 in HCC. We demonstrated that the expression level of MCM4 was negatively associated with the clinical stage and prognosis of HCC patients. Furthermore, we also revealed the functions of MCM4 in tumor behavior including tumor proliferation.

\subsection{Bioinformatics analysis}

The data from GTEx database and TCGA database were used for differential genetic analysis. Differential gene expression and survival analysis were measured using the GEPIA website (http://gepia.cancer-pku.cn) (11). One-way ANOVA was applied for gene expression analysis between cancer and non-cancerous liver tissues. The disease-free survival time and overall survival time were obtained from the TCGA public database.

\subsection{Patients and samples}

Following Institutional Review Board approval, 102 patients with hepatocellular carcinoma who have been proved by pathology were incorporated into the study. Fresh samples were collected just after surgery and fixed in $10 \%$ formalin before embedding in paraffin wax. Patients' clinical and pathological data including age, gender, number of tumor nodes, tumor sizes and AFP levels were identified. All samples used were analyzed by experienced pathologists to ensure they were diagnosed as HCC. Written informed consent was obtained from patients before the study. We have also complied with the World Medical Association

\section{Materials and Methods}


Declaration of Helsinki involving the ethical conduct of research involving human subjects.

\subsection{Immunohistochemistry}

A standard immunoperoxidase staining procedure was performed. The paraffin-embedded tissues were sliced into $4 \mathrm{um}$ sections and baked at $70^{\circ} \mathrm{C}$ for 45 minutes. The sections were de-waxed in xylene and rehydrated in graded ethanol. Then, $0.01 \mathrm{M}$ citrate buffer was applied to repair the antigen, $3 \% \mathrm{H}_{2} \mathrm{O}_{2}$ in methanol was added to the slices for 10 minutes. The tissues were incubated with primary anti-MCM4 antibodies (rabbit, Sigma-Aldrich) at a 1:500 dilution overnight. After washing with phosphate buffered saline, the tissues were incubated with goat anti-rabbit IgG at room temperature for 40 minutes. After DAB staining, the sections were counterstained with hematoxylin, dehydrated in ethanol, and cleared with xylene. For the results analysis, semiquantitative $\mathrm{H}$-score was computed for each sample by multiplying the staining intensities ( 0 : negative, 1 : weak staining, 2: moderate staining, 3: strong staining) and distribution areas (0-100\%). All samples were classified as high expression and low expression groups, respectively, according to the distribution of $\mathrm{H}$-score.

\subsection{Cell culture and transfection}

Both human hepatocellular carcinoma cell lines (Hep3B and SNU-475) used in this study were purchased from the Cell Bank of the Chinese Academy of Sciences (Shanghai, China). SNU-475 was cultured in RPMI1640 medium while Hep3B was cultured in Dulbecco's modified Eagle's medium (DMEM), both contained $1 \%$ penicillin streptomycin and $10 \%$ fetal bovine serum. Cells were cultured at $37^{\circ} \mathrm{C}$ with $5 \% \mathrm{CO}_{2}$. Both cell lines were maintained in our institution. For in vitro studies, both Hep3B and SNU-475 cells were transfected with shRNA to silence MCM4 according to the manufacturer's instructions. Short hairpin RNA (Target sequence: AAATGCATTCTTCAGCTATCCCT and AAATGTTGGCATAGATATTACTG) was obtained from the National Core Facility for Manipulation of Gene Function by RNAi, miRNA, miRNA sponges, and CRISPR/Genomic Research Center, Academia Sinica, Taipei, Taiwan. The stable cell lines were verified by RT-qPCR and Western blot before proceeding to the next experiment.

\subsection{RT-PCR}

The total RNA derived from cells were isolated using TRIzol $^{\circledR}$ reagent (Invitrogen; Thermo Fisher Scientific, Inc.) according to protocols. After measuring the content by ultraviolet analysis, cDNA was synthesized from total RNA applying Fast Reverse Transcriptase (Tiangen Biotech Co., Ltd.). Quantitative PCR was performed on a Smart Cycler using SGExcel FastSYBR Mixture (With Low ROX) Plus (Sango biotech, China). To further analyze the real-time PCR data, we applied a comparative threshold cycle $(\mathrm{Ct})$ method that compares differences in $\mathrm{CT}$ values between target RNA and common control (12). The forward and reverse primers are shown below (MCM4, F, 5'-TTGAAGCCATTGATGTGGAA-3' and R, 5'-GGCACTCATCCCCGTAGTAA-3'; GAPDH, F, 5'-GAGTCAACGGATTTGGTCGT-3' and R, 5'-TTGATTTTGGAGGGATCTCG-3').

\subsection{Western blot}

Cell samples were lysed in RIPA lysis buffer, and protein concentrations were determined using a BCA Protein Assay kit (Beijing Solarbio Science \& Technology Co., Ltd.). Then the denatured protein $(\sim 30 \mu \mathrm{g})$ was loaded and separated in SDS-PAGE gels and transferred to a wet polyvinylidene membrane. After blocking with 5\% dry milk for $1 \mathrm{~h}$ at $37^{\circ} \mathrm{C}$, primary antibodies including MCM4 (1:1,500 dilution, ab4461, Abcam), mouse anti- $\beta$ actin (1:1,000 dilution, ab8226, Abcam plc, Cambridge, UK) were added and incubated at $4^{\circ} \mathrm{C}$ overnight. After washing with TBS with Tween-20, the membranes were further incubated with HRP-conjugated goat anti-rabbit IgG antibody (Abcam, cat. no. ab181662, 1:2,000) at room temperature for $1 \mathrm{~h}$. The blots were visualized using an enhanced chemiluminescence detection kit (Thermo Fisher Scientific, Inc.).

\subsection{Colony formation array}

Hep3B and SNU-475 cells were seeded and cultured on $60 \mathrm{~mm}^{2}$ plates at an initial density of $800 /$ well, and each group was measured in 3 parallel wells. After 2 weeks, cells were washed and fixed with $10 \%$ formaldehyde for $15 \mathrm{~min}$ at room temperature. The cells were then stained with Giemsa for $15 \mathrm{~min}$. Colony numbers were counted by using an optical microscope.

\subsection{MTT assay}

Both Hep3B and SNU-475 cells were seeded and cultured on 96-well plates at a density of 3500 cells/well. The cell's proliferation capacity was measured by MTT (methyl thiazolyl tetrazolium) assay. $0.02 \mathrm{~mL}$ of $5 \mathrm{mg} / \mathrm{mL}$ MTT reagent was added into each well for 24 hours at $37^{\circ} \mathrm{C}$. The medium was replaced by $0.15 \mathrm{~mL}$ of dimethyl sulfoxide (DMSO, Sigma) for 10min incubation. A microplate spectrophotometer (Thermo Scientific, Franklin, MA) was used to measure the optical density at $570 \mathrm{~nm}$. All experiments were performed in triplicate.

\subsection{Tumorigenicity assay}

For in vivo xenograft studies, $1 \times 10^{7}$ Hep3B cells transfected with MCM4 shRNA and controls were 
subcutaneously injected into the left flank of 8week-old BALB/c nude mice (Slac Laboratory Animal Co. Ltd, Shanghai, China). The mice were euthanized 4 weeks postinjection, and the tumors were excised and fixed for subsequent histopathological examination and analysis. Meanwhile, tumor volumes were measured twice a week after two weeks, tumor volume $=1 / 2\left(\right.$ length $\times$ width $\left.^{2}\right)$. All animal experiments were approved by the Animal Care and Use Committee of Tianjin Medical University Cancer Institute \& Hospital.

\subsection{Statistical analyses}

SPSS.22.0 statistic software (IBM Corp.) was applied to analyze the data. All data are presented as the mean \pm SD. Student's $t$-test was used for continuous variables, $\chi^{2}$ tests were applied to analyze categorical variables. Survival of patients was plotted using Kaplan-Meier method. $P$-value was two-sided and $p<0.05$ was considered to indicate a statistically significant difference.

\section{Results}

3.1. MCM4 was highly expressed in hepatocellular carcinoma

To explore the potential roles of MCM4 in hepatocellular carcinoma, we first compared the mRNA expression of MCM4 in hepatocellular carcinoma and normal liver tissues by Bioinformatics analysis. Differential gene expression analysis was conducted from GTEx and TCGA database and 369 primary HCC samples and 160 normal liver tissues were analyzed. As Figure 1A shows, MCM4 was significantly overexpressed in HCC. Moreover, we further explored the expression of MCM4 in serval HCC cell lines through public dataset (CCLE). As Figure 1B shows, MCM4 represented a high expression level in all five HCC cell lines. Next, we investigated the expression of MCM4 protein in HCC and normal liver tissues from 102 patients via IHC. Similar to mRNA expression, the expression of MCM4 protein was significantly overexpressed in HCC (Figure 1). Moreover, the positive signal of MCM4 was mainly expressed in the nuclei of tumor cells and showed typical strong and weak staining (Figurer 1C). In contrast, normal liver tissues barely expressed MCM4 (Figure 1D).

3.2. High expression of MCM4 correlated with clinicopathological variables and prognosis of $\mathrm{HCC}$

To investigate the correlation between MCM4 protein expression status and clinical pathological characteristics of HCC patients, we divided HCC patients into two groups according to the expression of MCM4 protein. The associations between MCM4 protein expression
A

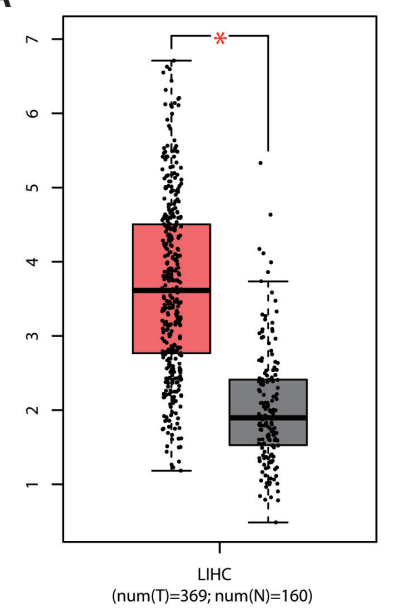

B

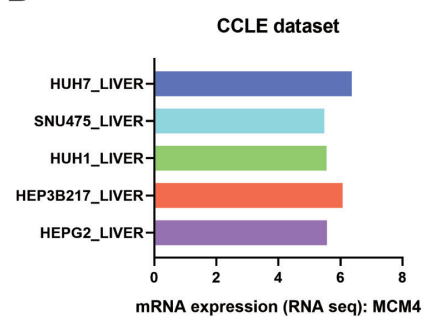

C

100
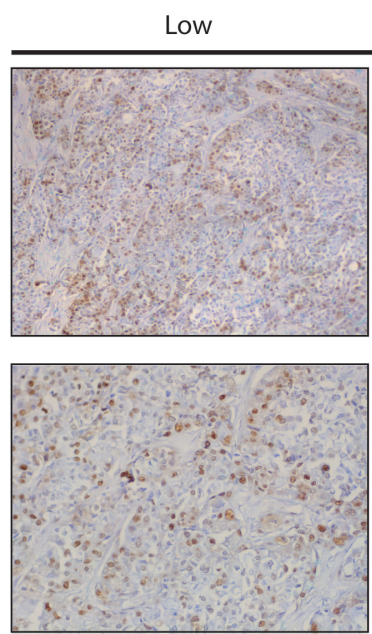

100

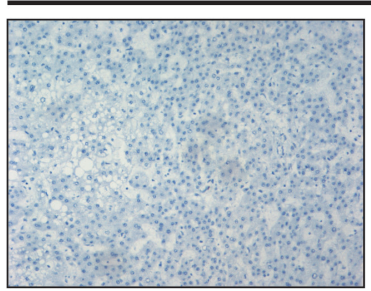

High
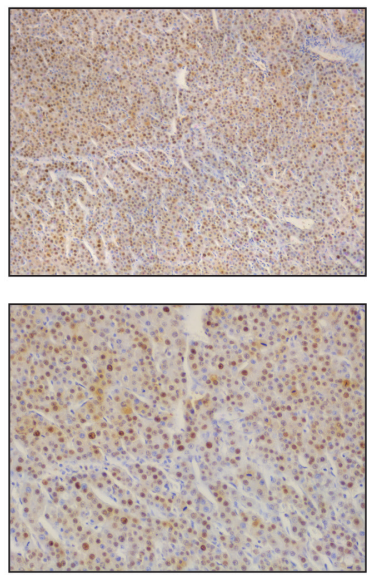

200

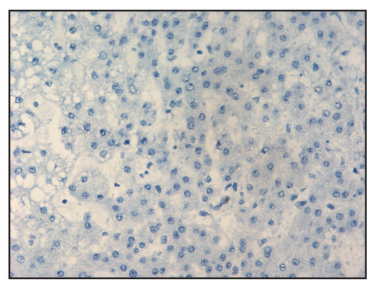

Figure 1. The expression of MCM4 in human HCC cell lines and tissues. (A) The expression of MCM4 mRNA level in HCC and normal liver tissues. (B) MCM4 mRNA expression in the CCLE dataset. (C) Immunostaining showed high and low expression of MCM4 in HCC tissues. (D) Immunostaining showed negative or weak expression of MCM4 in adjacent benign liver tissues. 
and clinicopathological features are shown in Table 1. High expression of MCM4 protein was significantly correlated with larger tumor size $(p=0.043)$. As for other clinicopathological features including age, gender, number of tumor nodes, and AFP level, no associations were found (all $p>0.05$ ). On the other hand, we assessed the association between MCM4 expression and prognosis to identify the prognostic value of MCM4 for HCC. Overall survival (OS) and disease-free survival (DFS) information were obtained from TCGA database. A significant correlation was found between MCM4 expression and adverse clinical outcomes including short OS and DFS of HCC patients (Figure 2A and 2B).

3.3. MCM4 promotes the proliferation of HCC cancer cells

To understand the potential roles of MCM4 in malignant behavior of $\mathrm{HCC}$, we inhibited the expression of MCM4 in HCC cancer cells. MCM4 expression was knocked down in both Hep3B and SNU-475 cells using shRNA. Both RT-PCR and Western blot results showed that shRNA worked as we expected, the expression of MCM4 was dramatically decreased in the shRNA group (Figure 3A and 3B).

Next, we tested whether loss-of-function of MCM4 is correlated with the proliferation of HCC cells by MTT assay and colony formation arrays. We found that downregulated expression of MCM4 significantly inhibits the proliferation of Hep3B and SNU-475 cells compared to controls $(p<0.05)$, manifested as decreased cell proliferation rate and colony formation (Figure 4A and 4B).

3.4. Downregulated expression of MCM4 suppressed the tumorigenicity of HCC cells in vivo

To further explore whether the role of MCM4 was

Table 1. Relationships of MCM4 and clinicopathological characteristics in 102 patients with hepatocellular carcinoma

\begin{tabular}{|c|c|c|c|c|c|}
\hline \multirow{2}{*}{ Feature } & \multirow{2}{*}{ All $(n=102)$} & \multicolumn{2}{|c|}{ MCM4 expression } & \multirow{2}{*}{$\chi^{2}$} & \multirow{2}{*}{$p$} \\
\hline & & Low $(n=46)$ & $\operatorname{High}(n=56)$ & & \\
\hline \multicolumn{6}{|l|}{ Age (year) } \\
\hline$<60$ & 64 & 25 & 39 & 2.527 & 0.112 \\
\hline$\geq 60$ & 38 & 21 & 17 & & \\
\hline \multicolumn{6}{|l|}{ Gender } \\
\hline Male & 56 & 29 & 27 & 2.243 & 0.134 \\
\hline Female & 46 & 17 & 29 & & \\
\hline \multicolumn{6}{|c|}{ Number of tumor nodes } \\
\hline Single & 44 & 20 & 24 & 0.004 & 0.950 \\
\hline Multiple $\geq 2$ & 58 & 26 & 32 & & \\
\hline \multicolumn{6}{|l|}{ Tumor size } \\
\hline$<5 \mathrm{~cm}$ & 40 & 23 & 17 & 4.088 & $0.043^{*}$ \\
\hline$\geq 5 \mathrm{~cm}$ & 62 & 23 & 39 & & \\
\hline \multicolumn{6}{|l|}{$\operatorname{AFP}(\mathrm{ng} / \mathrm{mL})$} \\
\hline$<50$ & 34 & 15 & 19 & 0.020 & 0.888 \\
\hline$\geq 50$ & 68 & 31 & 37 & & \\
\hline
\end{tabular}

A

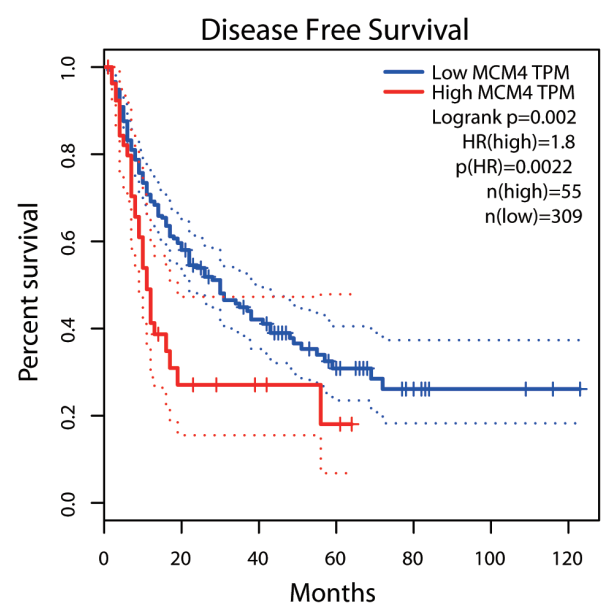

B

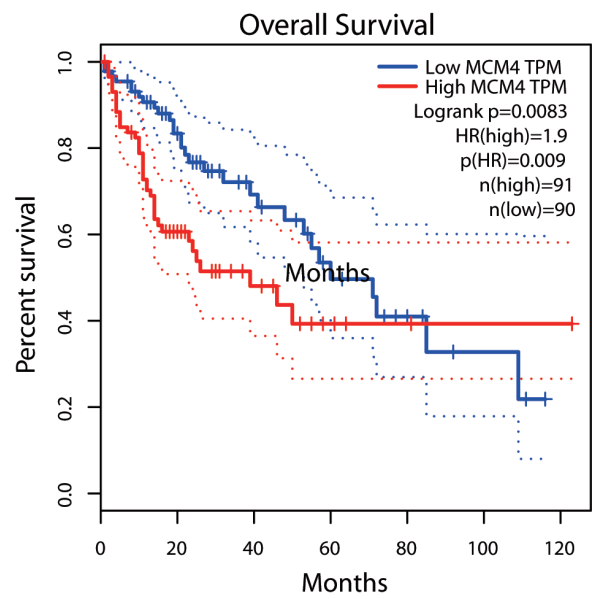

Figure 2. High expression of MCM4 as a prognostic factor of human HCC. (A) Kaplan-Meier survival analysis of disease-free survival for MCM4 expression in HCC. (B) Kaplan-Meier survival analysis of overall survival for MCM4 expression in HCC. 


\section{A}

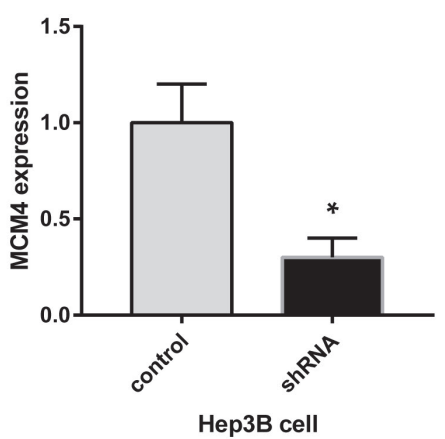

B

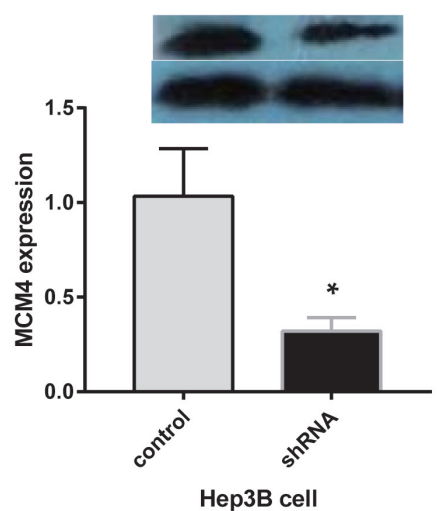

A

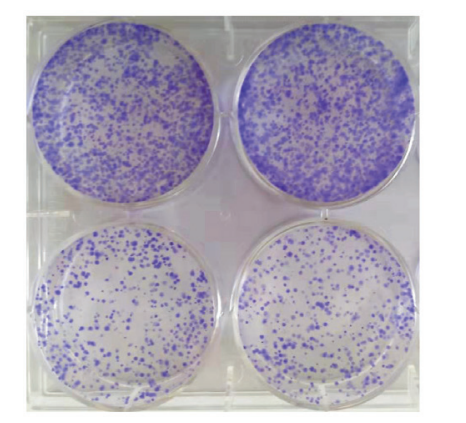

B

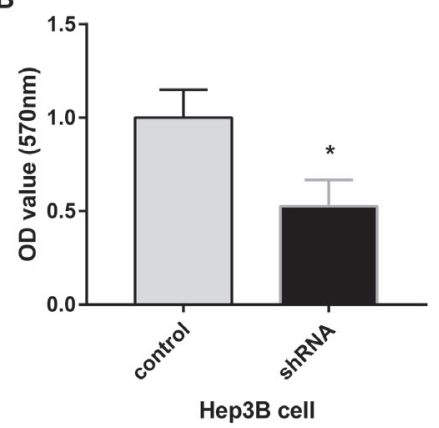

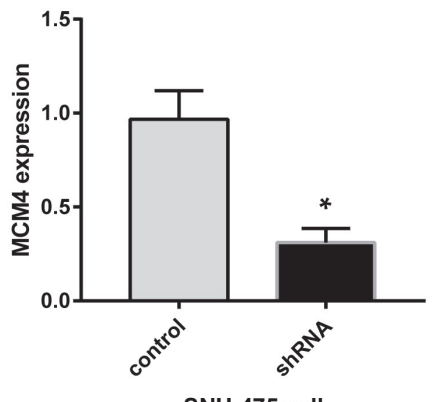

SNU-475 cell

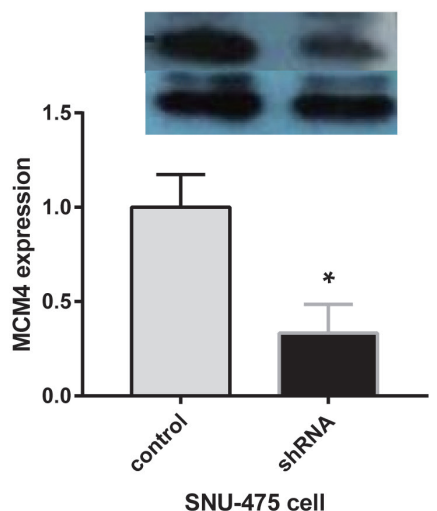

Figure 3. Stably knock down of MCM4 by shRNA in both Hep3B and SNU-475 cells. $(A, B)$ RT-PCR and Western blot showed lower expression level of MCM4 in shRNA group $(p<$ $0.05)$, compared to the control group. $(* p<0.05)$

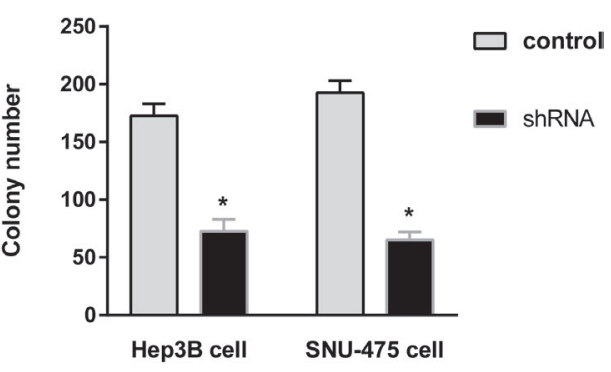

associated with abnormal in vitro behavior and could translate into abnormal tumorigenesis in vivo, cells from the MCM4 shRNA group and control group were injected subcutaneously into athymic mice respectively. The tumor volumes were measured twice a week after two weeks. As the growth curve shown in Figure 5A, tumor growth of the shRNA group was significantly slower than that of the control group ( $p$ $<0.05)$. Meanwhile, we also performed IHC to detect the expression of MCM4 in subcutaneous tumors. Consistently, MCM4 expression was dramatically decreased in the shRNA group, which indicated that an effective and stable knock-down of MCM4 was expressed in mouse tumors (Figure 5B). 


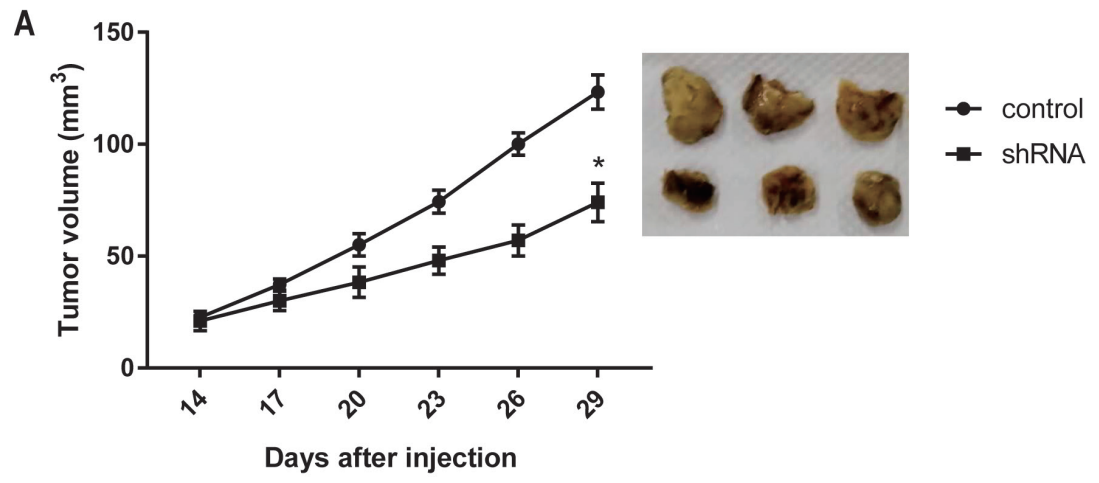

B

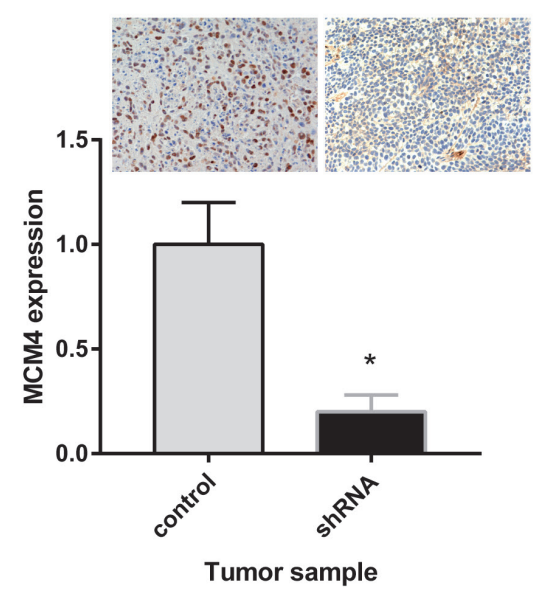

Figure 5. The influence of MCM4 on tumor growth of $\mathrm{HCC}$ in mice. (A) Representative images of tumors and tumor growth curves from both groups are shown. Tumor volumes of shRNA group were smaller than that of the control group $(p<0.05)$. (B) Immunohistochemistry demonstrated that the expression of MCM4 was dramatically decreased in mouse tumors $(p<0.05)$, which suggested a successful construction of MCM4 knock down model in mice. $\left({ }^{*} p<\right.$ $0.05)$

\section{Discussion}

In this study, we explored the potential role of MCM4 in HCC. We first showed that MCM4 was overexpressed in HCC tissues and high MCM4 expression represents a poor clinical outcome. Furthermore, we investigated the function of MCM4 in HCC aggression. Consistent with previous reports, MCM4 may be an effective marker and probably even a potential therapeutic target for malignant tumors, including HCC.

MCM4 is part of the MCM2-7 heterohexameric complex that is involved in origins of DNA replication prior to $S$ phase (8). Improper replication fork progression can lead to stalled forks, the potential for incomplete DNA replication and even fork collapse which may lead to double strand break (DSB) formation $(8,13)$. Therefore, the MCM proteins play important roles in maintaining genomic integrity. Deregulation of MCM proteins including MCM4 may contribute to cell proliferation and tumorigenesis. In our study, we demonstrated that MCM4 plays an important role in HCC cell proliferation. Knocking down MCM4 significantly decreased HCC cell proliferation rate and colony formation. Consistent with our findings, Choy $\mathrm{B}$ et al. investigated the correlation between MCM4 and MCM7 expression and Ki-67, Bmi1, and cyclin E expression in serval tumor types. They found that the percentage of MCM4 expression to be significantly correlated with Ki-67, Bmi1, and cyclin E expression

in esophageal carcinoma and precancerous lesions (3). Bagley et al. (8) identified a mutant allele of MCM4 in a spontaneous mouse model for dominantly inherited T-cell leukemia/lymphoma, and this MCM4 allele promoted the accumulation of focal chromosomal gains and losses, including aberration at the Notch1 locus that drives the formation of T-cell leukemia/lymphoma. Shima et al. (9) isolated a hypomorphic mutation of MCM4 in a phenotype-based screen for chromosome instability in mice and the mutation caused exclusively mammary adenocarcinoma in approximately $80 \%$ of homozygous females. They (14) also found that hypomorphic alleles of the genes encoding the subunits of the MCM2-7 complex may increase breast cancer risk.

The current status of HCC diagnosis and treatment urgently requires us to investigate more underlying mechanisms. Plenty of studies have reported that aberrant expression of MCM4 can be a promising prognostic marker and even a therapeutic target in a number of malignancies (4,6-10). In our study, the data from public databases suggested that high expression of MCM4 was correlated with clinical pathological characters such as tumor size. Moreover, a high level of MCM4 was associated with poorer OS and DFS of HCC patients. All these results indicated that MCM4 may be a potential prognostic predictor, which plays a great role in tumor progression. Similar to our results, Huang et al. reported that increased expression of MCM4 might 
be associated with pathological staging and it may be a valuable molecular marker involved in the development and/or genesis of esophageal cancer (10). Using public databases including TCGA and The Protein Atlas (TPA) database, Ahluwalia $\mathrm{P}$ et al. identified a novel 4 gene prognostic signature with clinical utility in colorectal cancer containing MCM4 (15). Moreover, a study also developed a four-gene predictive model of clinical responses to aromatase inhibitors in breast cancer patients by applying MCM4 (16).

In conclusion, we first showed the roles of MCM4 in HCC. Though more research is still needed to explore and verify the exact underlying mechanisms of HCC development and progression, we speculate that MCM4 can be a prognostic marker and even a therapeutic target for HCC.

Funding: This work was supported by the National Natural Science Foundation of China (No. 81471761, No.81501568) and the National Key Clinical Specialist Construction Programs of China (No. 2013-544).

Conflict of Interest: The authors have no conflicts of interest to disclose.

\section{References}

1. Yardimci H, Walter JC. Prereplication-complex formation: a molecular double take? Nat Struct Mol Biol. 2014; 21:20-25.

2. Forsburg SL. Eukaryotic MCM proteins: beyond replication initiation. Microbiol Mol Biol Rev. 2004; 68:109-131.

3. Choy B, LaLonde A, Que J, Wu T, Zhou Z. MCM4 and MCM7, potential novel proliferation markers, significantly correlated with $\mathrm{Ki}-67, \mathrm{Bmi1}$, and cyclin E expression in esophageal adenocarcinoma, squamous cell carcinoma, and precancerous lesions. Hum Pathol. 2016; 57:126-135.

4. Bochman ML, Schwacha A. The Mcm complex: unwinding the mechanism of a replicative helicase. Microbiol Mol Biol Rev. 2009; 73:652-683.

5. Giaginis C, Vgenopoulou S, Vielh P, Theocharis S. MCM proteins as diagnostic and prognostic tumor markers in the clinical setting. Histol Histopathol. 2010; 25:351-370.

6. Kikuchi J, Kinoshita I, Shimizu Y, Kikuchi E, Takeda K, Aburatani H, Oizumi S, Konishi J, Kaga K, Matsuno Y, Birrer MJ, Nishimura M, Dosaka-Akita $\mathrm{H}$. Minichromosome maintenance $(\mathrm{MCM})$ protein 4 as a marker for proliferation and its clinical and clinicopathological significance in non-small cell lung cancer. Lung Cancer. 2011; 72:229-237.
7. Ladstein RG, Bachmann IM, Straume O, Akslen LA. $\mathrm{Ki}-67$ expression is superior to mitotic count and novel proliferation markers $\mathrm{PHH} 3, \mathrm{MCM} 4$ and mitosin as a prognostic factor in thick cutaneous melanoma. BMC Cancer. 2010; 10:140.

8. Bagley BN, Keane TM, Maklakova VI, Marshall JG, Lester RA, Cancel MM, Paulsen AR, Bendzick LE, Been RA, Kogan SC, Cormier RT, Kendziorski C, Adams DJ, Collier LS. A dominantly acting murine allele of Mcm4 causes chromosomal abnormalities and promotes tumorigenesis. PLoS Genet. 2012; 8:e1003034.

9. Shima N, Buske TR, Schimenti JC. Genetic screen for chromosome instability in mice: $\mathrm{Mcm} 4$ and breast cancer. Cell Cycle. 2007; 6:1135-1140.

10. Huang XP, Rong TH, Wu QL, Fu JH, Yang H, Zhao JM, Fang Y. MCM4 expression in esophageal cancer from southern China and its clinical significance. J Cancer Res Clin Oncol. 2005; 131:677-682.

11. Tang Z, Li C, Kang B, Gao G, Li C, Zhang Z. GEPIA: a web server for cancer and normal gene expression profiling and interactive analyses. Nucleic Acids Res. 2017; 45:W98-W102.

12. Livak KJ, Schmittgen TD. Analysis of relative gene expression data using real-time quantitative PCR and the 2(-Delta Delta C(T)) Method. Methods. 2001; 25:402408.

13. Allen C, Ashley AK, Hromas R, Nickoloff JA. More forks on the road to replication stress recovery. J Mol Cell Biol. 2011; 3:4-12.

14. Shima N, Alcaraz A, Liachko I, Buske TR, Andrews CA, Munroe RJ, Hartford SA, Tye BK, Schimenti JC. A viable allele of $\mathrm{Mcm} 4$ causes chromosome instability and mammary adenocarcinomas in mice. Nat Genet. 2007; 39:93-98.

15. Ahluwalia P, Mondal AK, Bloomer C, Fulzele S, Jones K, Ananth S, Gahlay GK, Heneidi S, Rojiani AM, Kota V, Kolhe R. Identification and clinical validation of a novel 4 gene-signature with prognostic utility in colorectal cancer. Int J Mol Sci. 2019; 20.

16. Turnbull AK, Arthur LM, Renshaw L, Larionov AA, Kay C, Dunbier AK, Thomas JS, Dowsett M, Sims AH, Dixon $\mathrm{JM}$. Accurate prediction and validation of response to endocrinetherapy in breast cancer. J Clin Oncol. 2015; 33:2270-2278

Received January 10, 2021; Revised February 25, 2021; Accepted March 3, 2021.

*Address correspondence to:

Zhi Guo, Department of Interventional Therapy, Tianjin Medical University Cancer Institute \& Hospital, Huanhuxi Road, Hexi District, Tianjin 300060, China.

E-mail: guozhi9960@sina.com

Released online in J-STAGE as advance publication March 12, 2021. 